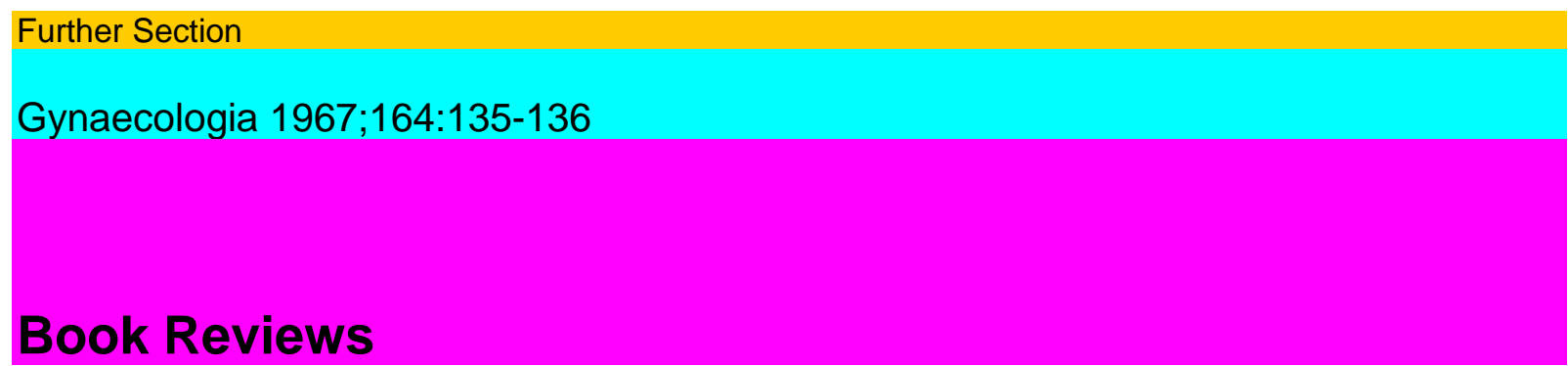

\title{
BUCHBESPRECHUNGEN LIVRES NOUVEAUX
}

Berger, J.: Die Fluoreszenzmikroskopie in der Früherfassung der weiblichen Genital -karzinome. Bibl. gynaec. Fasc. 43. Fortschritte der Geburtshilfe und Gynäkolo-gie. Vol. 30. Karger, Basel/New York 1967. 50 S., 18 Abb., 17 Tab., 2 Tafeln. Preis: Fr./DM 18.-.

Der Autor hat sich die Aufgabe gestellt, die Leistungsfähigkeit der Fluoreszenz-mikroskopie im Rahmen der Früherfassung der weiblichen Genitalkarzinome abzu-klären.

Nach kurzer Besprechung der Entwicklung und bisherigen Anwendungsbereiche der Fluoreszenzmikroskopie werden die allgemeinen physikalisch-chemischen Eigen-schaften fluoreszierender Substanzen dargelegt. Es folgt eine eingehende Erörte-rung des Akridinorange, wobei die Abhängigkeit der Fluoreszenz von der Konzen-tration der Farblösung, bzw. von deren Absorption im Gewebe, vom $\mathrm{pH}$, von der chemischen Zusammensetzung der

Verdünnungsflüssigkeit, von der Dauer der Farb-stoíFeinwirkung und der UV-Anregung aufgezeigt wird.

Die Untersuchungen wurden zunächst am normalen mehrschichtigen Platten-epithel der Portio durchgeführt, wo in Grundversuchen das unterschiedliche Ver-halten von Basal- bzw. Parabasalzellen einerseits und Oberflächen- bzw. Inter-mediärzellen anderseits festgestellt werden konnte. Mit zunehmender Epitheldiffe-renzierung ergab sich eine Abnahme der Orangefärbung. Die Technik der Fluoro-chromierung und die der Fluoreszenzmikroskopie werden detailliert beschrieben.

Von besonderem Interesse sind die Ergebnisse der Fluoreszenzmikroskopie beim einfachen und beim gesteigert atypischen Epithel sowie schliesslich beim invasiven Plattenepithelkarzinom. Es wurde beobachtet, dass in bestimmten $\mathrm{pH}$-Bereichen die Farbreaktionen bei den verschiedenen Epithelarten sich unterschiedlich ver-halten, und das Regenerationsepithel, welches aus kolposkopischen Umwandlungs-zonen gewonnen wurde, eine ähnliche Sekundärfluoreszenz aufweist wie das ein-fach atypische Epithel. Invasive Karzinome mit einer Vermehrung der RNS zeigen - infolge Affinität des Akridinorange zu den Ribonukleoproteiden - eine verstärkte Orangefluoreszenz.

Zusammenfassend wird in bezug auf die Fluorochromierung liistologischer Prä-parate hervorgehoben, dass mit einem einzigen Farbstoff, dem Akridinorange, eine polychromatische Darstellung der verschiedenen Schichten und einzelner Struktu-ren des Plattenepithels erfolgt, wobei sich verwertbare Unterschiede zwischen nor-malem, atypischem und karzinomatösem Gewebe ergeben.

Im letzten Abschnitt wird über die Erfahrungen mit dem Fluorochrom Akridinorange in der Zytologie berichtet.

Nach Darlegung der Technik und der zytochemischen Grundlagen der Fluorochromierung von Zellausstrichen werden die Kriterien für die Diagnose «Tumor-zelle» erläutert. Es wird dabei betont, dass es unzulässig ist, allein auf Grund einer orangeroten Fluoreszenz eine Tumordiagnose zu stellen, weil zunächst die verschiedenen physiko-chemischen 
Voraussetzungen zu berücksichtigen sind und ausserdem zu beachten ist, dass auch gutartige Zellen einen hohen RNS-Gehalt aufweisen können.

Der Vergleich der zytodiagnostischen Ergebnisse nach Papanicolaou- bzw. Akri-dinorangeFärbung zeigt, dass die Fluoreszenzmikroskopie als wertvolle Ergänzung

136 Book Reviews - Buchbesprechungen - Livres Nouveaux

zur erstgenannten Untersuchungsmethode gewertet werden darf, indem sie zur Klärung suspekter Befunde beizutragen vermag und die Erkennung der Adeno-karzinome erleichtert.

Es ist festzustellen, dass der Autor der vorliegenden Monographie seine Erfah-rungen sachlich darlegt und die Fluoreszenzmikroskopie auf Grund krilisch gesich-teter eigener Beobachtungen beurteilt. Die graphische Aufzeichnung der Befunde und die Wiedergabe anschaulicher, zum Teil mehrfarbiger Mikrophotographien er-gänzen den Text in sehr vorteilhafter Weise.

J. Jeder

der sich mit den Problemen der histologischen und zytologischen Diagno-stik des weiblichen Genitalkarzinoms befasst

wird aus der Lektüre der vorliegenden Arbeit von Bßrger grossen Nutzen ziehen. D. Da Rugna Basel 